\title{
Research on ordered charge and discharge of cluster electric vehicle based on index selection
}

\author{
Chunxuan $\mathrm{Hu}^{*}$, Tianran Li, and Chao Yuan \\ Nanjing Normal University NANRUI Electric and Automation College, Nanjing, China
}

\begin{abstract}
The basic characteristics of electric vehicles are important basis for studying the behavior of electric vehicles. According to the basic characteristics of electric vehicles, this paper establishes an electric vehicle convergence model and its control strategy with demand-side response. Taking into account the demand for electric vehicles, electric vehicle aggregators and power companies, reducing the cost of control, while reducing the impact on electric vehicles. Based on the real-time state of charge, the conditions of electric vehicle in the network and other factors to build the assessment model of the scheduling potential, and then put forward the demand response indicators of electric vehicles, and give the corresponding aggregation strategy. considering the multiple constraints, such as the cost constraints of electric vehicles participating in grid regulation, the charging requirements of electric vehicle owners, and the battery consumption of electric vehicles, a control strategy model is proposed for electric vehicles participating in demand response of power systems. The simulation test shows that the aggregation strategy can not only meet the travel needs of electric vehicle owners, but also reduce the impact on the electric vehicle caused by frequent switching of charge and discharge status. In addition, it can also reduce the cost of grid regulation.
\end{abstract}

\section{Introduction}

In recent years, electric vehicles have developed rapidly as new types of transportation, but they have also brought new challenges to the safety and management of power grids[1-3]. If a large-scale disorderly electric vehicle is integrated based on different time and scheduling orders, and used it as a demand-side response resource that can be flexibly scheduled. which can not only eliminate adverse impacts, but also can be used to suppress load and Renewable energy fluctuations, frequency modulation and other functions effectively support grid operation[4]. Literature[5] divide electric vehicles into several electric car clusters with different priorities to solve the large-scale real-time optimization scheduling problem according to different charging completion time of accessing electric vehicles, Using the Grey Wolves algorithm (GWO) and the energy buffering consensus algorithm to solve the charge and discharge problems inside and outside the electric vehicle cluster, the problem of energy redistribution within the cluster can be better resolved, but the participation of the electric vehicle in regulating electricity price and grid electricity

\footnotetext{
* Corresponding author: strawberry_hcx@163.com
} 
price constraints are not considered in the cluster division. Reference [6] considers electric vehicles as a component that can provide spare capacity for the power grid, but the capacity that this component can provide affected by many factors. Based on this problem, it proposes an evaluation algorithm include real-time dispatchable capacity of scaled electric vehicles, but its study did not take other important factors into account. At present, many reference regard the direct dispatching of electric vehicles as the basic assumption[7-10]. In fact, the dispatching organization does not have this authority, and the dispatching institution directly schedules each electric car that is connected to it, the problem of dimensional disaster and long calculation time may occur.

\section{Establishment of a single electric vehicle charging model}

\subsection{Electric vehicle's state of charge}

Assuming that the power consumption of the electric vehicle when it consumes electric energy and supplementing electric energy is constant, the state of charge of the on-board battery can be calculated according to the basic model of ordinary battery charge and discharge:

$$
S O C_{i}(t+\Delta t)=\mp P_{(c, f), i} \eta_{(c, f), i} \Delta t / C_{i}+S O C_{i}(t)
$$

In the formula above, $S_{S O}(t+\Delta t)$ is the state of charge of electric vehicle $i$ at time $(t+\Delta t), S O C_{i}(t)$ is the state of charge of electric vehicle $i$ at time $t, P_{c, i}$ is the charging power of electric vehicle $i$, and $\eta_{c, i}$ is the charging efficiency of electric vehicle $i, P_{f, i}$ is the discharge power of the electric vehicle $i$, and $\eta_{f, i}$ is the discharge efficiency of the electric vehicle $i$. The negative sign is taken when discharging. $C_{i}$ is the battery capacity of the electric vehicle $i$, and $\Delta t$ is the time interval.

\subsection{Schedulable mode decision}

Through the real-time SOC of the electric vehicle battery, the current charging and discharging dispatching potential can be obtained to the greatest degree. Despite the constraints of travel planning, the relationship between the SOC of the electric vehicle and its dispatchable potential is first considered here. According to the SOC of the electric vehicle at time $t$, it can be divided into the following three categories:

\section{Electric vehicle regulation index}

Electric vehicle aggregators aggregate a certain number of electric vehicles to manage them collectively, and use the demand response to participate in the regulation of the electric power system to obtain economic benefits. Based on factors such as battery life, controllable state, state of charge, economic effect, etc., this paper proposes four kinds of electric vehicle control order indicators and comprehensive judgment indicators for aggregators to select suitable electric vehicles to participate in scheduling at different times.

\subsection{State switching index $f_{1}$}

In order to prolong battery life, try not to let the electric car switch frequently. Setting the index $f_{1}$ to measure the frequency of electric vehicle state switching: 


$$
f_{1}=\left\{\begin{array}{c}
0, t_{i} \leq t_{\text {limit }} \\
\frac{t_{\text {dura }}}{t_{\text {dura }}^{\text {max }}}, t_{i}>t_{\text {limit }}
\end{array}\right.
$$

In the above equation, $t_{\text {dura }}$ indicates the time interval between the shift of an electric vehicle from one state to another, and $t_{d u r a}^{\max }$ indicates the maximum interval time between the shift of a controllable electric vehicle from one state to another. $t_{\text {limit }}$ is the set minimum switching time. $t_{i}$ denotes the time interval between the two states of the $i$-th electric vehicle. It is easy to get $f_{1} \in[0,1]$. The bigger $f_{1}$, the more priority it should be to control.

\subsection{Control coefficient index $\mathbf{f}_{2}$}

The index $f_{2}$ indicates the controllability of the electric vehicle:

$$
f_{2}=\left\{\begin{array}{cl}
0 \quad, & \frac{t_{o f f}^{i}-t_{o n}^{i}}{T_{M I N}^{i}} \leq 1 \\
\frac{t_{o f f}^{i}-t_{o n}^{i}}{T_{M I N}^{i}}=\frac{T_{S E T}^{i}}{T_{M I N}^{i}}, & \frac{t_{o f f}^{i}-t_{o n}^{i}}{T_{M I N}^{i}}>1
\end{array}\right.
$$

In the formula, $t_{o n}^{i}, t_{o f f}^{i}$, respectively, the time when the $i$-th vehicle is connected to the grid and the pre-set stop charging time of the vehicle owner are respectively based on the requirements of the automobile owner. The shortest charging time requirement for charging the $i$-th car by $T_{M I N}^{i}$ :

$$
T_{M I N}^{i}=\frac{\left(S O C_{E N D}^{i}-S O C_{O N}^{i}\right)}{\eta_{i} P_{N}^{i}} C_{B}^{i}
$$

In the formula, $P_{N}^{i}$ is the rated charging power of an electric vehicle. $C_{B}^{i}$ indicates the battery capacity of the electric vehicle; the SOC state of the electric vehicle just after being connected to the grid is represented as $S O C_{O N}^{i}$, the $S O C$ state at the time of leaving the grid is indicated as $S O C_{E N D}^{i}$, and $\eta_{i}$ indicates the charging and discharging efficiency of the electric vehicle.

If the defined charging time $T_{S E T}^{i}=\left(t_{o f f}^{i}-t_{o n}^{i}\right) \leq T_{M I N}^{i}$, the electric vehicle has been charged in the grid with the rated power $P_{N}^{i}$ until it leaves the grid.

\subsection{State of charge indicator $\mathbf{f}_{3}$}

The indicator $f_{3}$ represents the current state of charge (SOC) of an electric vehicle. According to two different conditions of charge and discharge, $f_{3}$ is expressed as:

$$
f_{3}=\left\{\begin{array}{l}
\frac{S O C(t)}{S O C_{\max }}, P_{\text {reg }}(t)<0 \\
\frac{1-S O C(t)}{1-S O C_{\min }}, P_{\text {reg }}(t)>0
\end{array}\right.
$$

In the formula, $S O C_{\max }$ and $S O C_{\min }$ are the maximum and minimum values of the state of charge of the electric vehicle at the current moment, and it is easy to know that $f_{3}$ is a number between 0 and 1 , and is a time variable. Where $P_{r e g}(t)=P_{d}(t)-P_{0}(t)$, the natural charging power of the electric vehicle cluster at time $t$ is $P_{0}(t)$. Let $P_{d}(t)$ represent the power tracking sent by the upper grid control center to the cluster electric vehicle. aims. When $P_{\text {reg }}(t)>0$, it means that $P_{d}(t)>P_{0}(t)$, that is, the natural charging power of the cluster electric vehicle is smaller than the control target that needs to be achieved at this time, and the whole cluster needs to increase the charging power; when $P_{\text {reg }}(t)<0$, it indicates that the electric vehicle cluster needs to increase the discharge power. 


\subsection{Economic Indicators $\mathbf{f}_{4}$}

The index f 4 indicates the content related to economic benefits. If the electric vehicle puts the unit price of electricity to the grid more than the marginal cost of the additional electricity generated by the grid itself, then the grid is selected to increase the power rather than let the electric vehicle participate in the regulation. F_4 Select whether existing electric vehicles are involved in regulation and control:

$$
f_{4}=\left\{\begin{array}{c}
0 \quad, \text { Price }_{\text {car }}^{i} \geq \text { Price }_{\text {marginal }} \\
\frac{\text { Price }_{\text {marginal }}-\text { Price }_{c a r}^{i}}{\text { Price }_{\text {marginal }}}, \text { Pric }_{\text {car }}^{i}<\text { Price }_{\text {marginal }}
\end{array}\right.
$$

In the formula, Price marginal represents the required cost price of the power generation unit of the grid, Price ${ }_{c a r}^{i}$ represents the price of the electric energy of the unit electric quantity of the i-th electric vehicle. In theory, because each electric vehicle has a different owner, so $P_{c a r}^{i}$ changes according to the change of the vehicle. At the same time, Price $e_{c a r}^{i}$ should also be a time variable Price $_{c a r}^{i}(t)$. The regulation costs required by the owners to participate in the regulation at different time periods are also different.

\subsection{Comprehensive judgment index}

Based on the above-mentioned indicators of regulatory order, comprehensive indicators are established to determine priorities:

$$
F=f_{2} f_{4}\left(\omega_{1} f_{1}+\omega_{2} f_{3}\right)
$$

In the formula, $\omega_{1}$ and $\omega_{2}$ represent the weights of the indices $f_{1}$ and $f_{3}$, respectively. The aggregator uses the comprehensive index $F$ to prioritize the regulated state of the electric vehicles that it manages, in order to minimize the number of electric vehicles participating in regulation and to reduce the impact of power system regulation on electric vehicle users.

\section{Analysis of examples}

\subsection{Simulation Scene Settings}

The scale of 5000 electric vehicle clusters in the industrial and commercial area is selected as the research object. The time $t_{\text {on }}$ of the cluster electric vehicle network is subject to the normal distribution of $N(8.5,2)$, and the off-net time is subject to the normal distribution of $N(18,2)$; Assuming the same EV type, the battery capacity is $48 \mathrm{KWh}$, the charging power limit is $8 \mathrm{KW}$, the discharge power limit is $-8 \mathrm{KW}$, the charging pile efficiency is 0.95 ; set the initial SOC to obey $N(0.5,1)$ normal distribution, the maximum $S O C_{\max }$ is 1 , the minimum $S O C_{\text {min }}$ is 0.2 ; Select marginal price $P_{\text {marginal }} 0.8$ yuan/degree, $P_{\text {car }}$ obey normal distribution of $N(0.6,1)$; take $\omega_{1}=0.5, \omega_{2}=0.5$; suppose $85 \%$ of owners with all $\mathrm{F}$ values greater than 1 are willing to participate in regulation; The owner of the vehicle is considered to have full autonomy over whether or not his electric vehicle is involved in the regulation and the time and departure time of the car's access to the power grid; it is assumed that the vehicle owner will not change the driving arrangement of the electric vehicle after the electric vehicle is connected to the grid. A typical workday EV driving state was used for simulation. The simulation period was $\Delta t=15 \mathrm{~min}$. The whole day was divided into 96 periods at $24 \mathrm{~h}$, and the first time point was $7 \mathrm{am}$. It is considered that the basic conditions of electricity consumption during each time period are unchanged, that is, the normal load of the grid and the charge and discharge load of the electric vehicle are not 
changed. In the grid, regular peak load is generated from 9:30am to 11:45am (the 10th to the 19th) in the morning, and the second peak occurs in the evening from 18:30 to 20:15 (the 46th to the 53th time).

\subsection{Ordered Charge Simulation}

The power values that need to be adjusted at different times of the power grid can be obtained. Table 1 shows that:

Table 1. Peak cutting and filling.

\begin{tabular}{|c|c|}
\hline Time & Peak clipping/filling \\
\hline $1-9$ & Peak clipping \\
\hline $10-63$ & filling \\
\hline $63-96$ & Peak clipping \\
\hline
\end{tabular}

According to the research data of the "Beijing Traffic Development Annual Report 2016"[11], Figure 1 can be obtained.

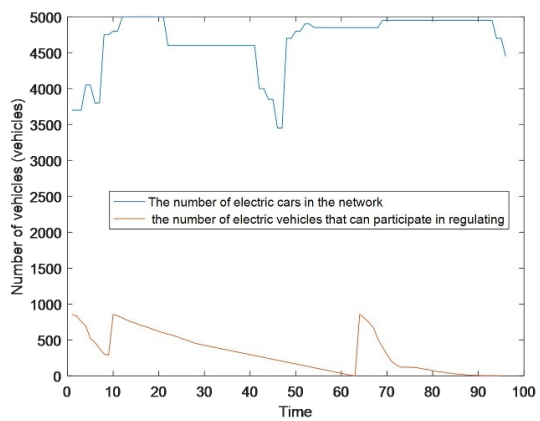

Fig. 1. Changes in the number of electric vehicles and the number of controllable electric vehicles in the network at different times.

Figure 1 shows the curve of the number of electric vehicles in the network and the number of controllable electric vehicles at different times. The number of electric vehicles in the network is simulated based on the data provided in document 11 , and the changes are calculated based on the current status of electric vehicles. The number of controllable electric cars. It can be seen from Fig. 2 that the regulation capacity provided by electric vehicles can meet the regulation and command of the grid, and only a slight deviation appears at the peak time. According to Fig. 3, it can be seen that the regulation capacity provided by the electric vehicle cluster is in a complementary relationship with the normal load of the grid, and the effect of the smooth grid load curve can be achieved.

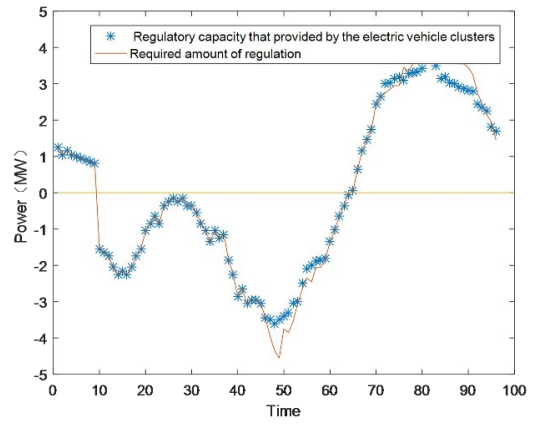

Fig. 2. Electric vehicle clusters provide regulatory capacity.

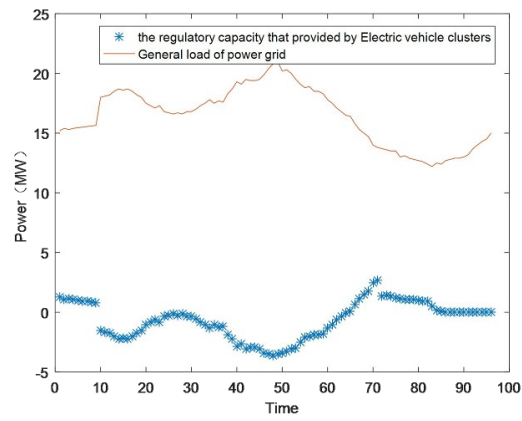

Fig. 3. Electric vehicle cluster participates in grid regulation. 


\section{Concluding}

In this paper, a demand-side response is proposed based on the index method of the electric vehicle convergence model, which can be considered according to the indicators such as the frequency of switching from one state to another state of the electric vehicle, the current SOC of the vehicle, whether it is controllable, and the control cost. Grid companies can manage load curves by adopting control measures to achieve the effect of peak-shaving and valley-filling, which will help alleviate the power imbalance in the power system. At the same time, this control strategy also takes into account the needs of the electric vehicle itself and the owner, which not only can meet the travel needs of electric vehicle owners, but also reduce the impact on the electric vehicle caused by the frequent switching of the charge and discharge status, and can also reduce the regulatory costs of power grid.

\section{References}

1. CLEMENT-NYNS K,HAESEN E,DRIESEN J. The impact of charging plug-in hybrid electric vehicles on a residential distribution grid[J].IEEE Trans on power Systems,2010,25(1):371-380.

2. LI Huiling,BAI Xiaomin. Influence of Electric Vehicle Charging on Distribution Network and Countermeasures [J]. Automation of Electric Power System, 2011,35(17):38-43.

3. XU Lizhong,YANG Guangya,XU Zhao, F.MARRA,C.TRHOLT. Influence of Electric Vehicle Charging Load on Danish Distribution System[J]. Automation of Electric Power System, 2011,35(14):18-23.

4. HU Zechun,SONG Yonghua,XU Zhiwei,LUO Zhuowei,ZHAN Kaiqiao,JIA Long. Influence and Utilization of Electric Vehicles' Access to Power Grid[J]. Proceedings of the CSEE, 2012,(04):1-10+25.

5. PAN Zhenning,ZHANG Xiaoshun,YU Tao,WANG Dezhi. Hierarchical real-time optimization scheduling of large-scale electric vehicle clusters[J]. Automation of Electric Power System, 2017,(16):96-104.

6. ZHANG Cong,ZHANG Xiangwen,XIA Junrong,SUN Haishun,WANG Chun,XU Xiaohui,WU Ke. Study on Real-time Scheduling Capacity Evaluation Method for Electric Vehicles[J]. Power System Protection and Control, 2015,43(22):99-106.

7. ZHANG Xinsong,Gu Juping,YUAN Yue,WANG Min,GUO Xiaoli,WANG Jianping. Electric Vehicle Optimal Charging Model and Its Approximate Solution Method[J]. Proceedings of the CSEE, 2014,(19):3148-3155.1

8. XIN Hao,YAN Zheng,XU Shaolun. Electric vehicle coordinated charging strategy based on multi-agent system[J]. Power System Technology, 2015,(01):48-54.

9. YAO Weifeng,ZHAO Junhua,WEN Fushuan,XUE Yusheng,XIN Jianbo. Dual-level optimization based charging and discharging scheduling strategy for electric vehicles[J]. Automation of Electric Power System, 2012,(11):30-37.

10. Liu H, Hu Z C, Song Y H, et al. Vehicle-to-Grid Control for Supplementary Frequency regulation Considering Charging Demands $[\mathrm{J}]$. IEEE Transactions on Power Systems, 2015, (99):1-9.

11. Beijing Transportation Development Research Institute. 2016 Beijing Transportation Development Annual Report [R], Beijing Institute of Transportation Development, 2016. http://www.bjtrc.org.cn/JGJS.aspx?id=5.2\&Menu=GZCG 